\title{
Studies of $\mathbf{B}_{s}$ Oscillations at LEP
}

\section{Christian Weiser*}

Universität Karlsruhe

Institut für Experimentelle Kernphysik

E-mail: 'Christian. Weiser@cern.ch'

Abstract: A review of $B_{s} \bar{B}_{s}$ mixing results from LEP is given, putting emphasis on recent measurements presented for the first time at this conference. Combined results from the B Oscillations Working Group are also presented. The LEP experiments exclude $\Delta m_{s}<14.3 \mathrm{ps}^{-1} @ 95 \% \mathrm{CL}$ with a sensitivity of $15.4 \mathrm{ps}^{-1}$. Combination of all available data on $B_{s}$ oscillations gives $\Delta m_{s}>14.6 \mathrm{ps}^{-1} @ 95 \% \mathrm{CL}$ with a sensitivity of $18.3 \mathrm{ps}^{-1}$.

\section{Introduction}

In the Standard Model, oscillations of neutral B mesons are described by second order weak interaction box diagrams with dominating contributions from top exchange. The mass difference of the mass eigenstates of $B_{d}$ and $B_{s}$ mesons is given by $\Delta m_{d, s}=\frac{G_{F}^{2}}{6 \pi^{2}}$. $m_{B_{d, s}^{0}} \cdot m_{t}^{2} \cdot F\left(\frac{m_{t}^{2}}{m_{w}^{2}}\right) \cdot\left|V_{t b}^{*} V_{t d, t s}\right|^{2} \cdot \eta_{b} \cdot f_{B_{d, s}}^{2} \cdot B_{B_{d, s}}$, thus allowing in principle the extraction of $V_{t d}$ from existing precise measurements of $\Delta m_{d}$. However, the non-perturbative parameters $f_{B}$ and $B_{B}$ have large theoretical uncertainties. The ratio $\frac{\Delta m_{s}}{\Delta m_{d}}=\frac{m_{B_{s}}}{m_{B_{d}}} \cdot \xi^{2} \cdot\left|\frac{V_{t s}}{V_{t d}}\right|^{2}$ is much better under control because many hadronic uncertainties cancel: $\xi^{2}=1.14 \pm 0.06$ [i]i]. $\left|V_{t d}\right|=A \lambda^{3} \sqrt{(1-\rho)^{2}+\eta^{2}}$ and $\left|V_{t s}\right|=$ $A \lambda^{2} \approx\left|V_{c b}\right|$ in the Wolfenstein parametrisation [2]. Since $\left|V_{t s}\right|$ does not depend on $\rho$ or $\eta, \Delta m_{s} / \Delta m_{d}$ and $\Delta m_{d}$ give the same kind of measurement or constraint on the unitarity triangle (especially on the angle $\gamma$ ) in the $\rho-\eta$ plane (Figure $\left.]_{1}^{1}\right)$. This is the main motivation to measure $B_{s}$ oscillations. One can deduce a probability density function for $\Delta m_{s}$ in the framework of the Standard Model from the other quantities con-

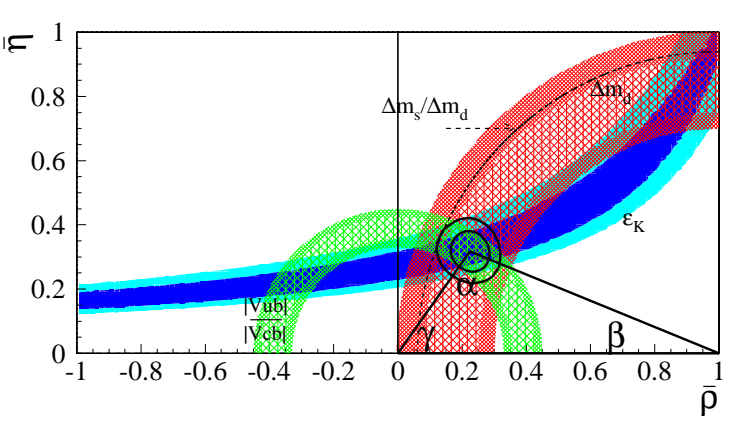

Figure 1: The various constraints on the unitarity triangle (from [i]1ㄱ) straining the unitarity triangle, giving expectation intervals $\Delta m_{s}=(16.3 \pm 3.4) \mathrm{ps}^{-1}$ or $\Delta m_{s}<23.2 \mathrm{ps}^{-1} @ 95 \%$ CL i i i

\footnotetext{
${ }^{*}$ Speaker.
} 
The time dependence of the mixing, neglecting $\mathrm{CP}$ violation and the width difference $(\Delta \Gamma \ll \Delta m)$, is for an initial $B^{0}$ state given by $P_{B^{0} / \bar{B}^{0}}(t)=\frac{\Gamma}{2} \cdot e^{-\Gamma t} \cdot[1 \pm \cos \Delta m \cdot t]$. Since $\Delta m_{s} / \Delta m_{d} \propto 1 / \lambda^{2} \approx 20$, very fast oscillations are expected for $B_{s}$ mesons. Typically $B_{s}$ mesons undergo many oscillations before they decay, making time integrated measurements impossible. Excellent time resolutions are thus essential to successfully measure $B_{s}$ oscillations. Another reason why $B_{s}$ oscillation analyses are so difficult, is the low fraction of $B_{s}$ mesons produced in $\mathrm{Z}$ decays: $f_{B_{s}}=(9.9 \pm 1.1) \%\left[\begin{array}{l}3 \\ 3\end{array}\right]$.

The significance of an oscillation signal is given by $\frac{1}{\sigma_{A}} \approx \sqrt{\frac{N}{2}} \cdot P_{s} \cdot(1-2 \eta) \cdot e^{-\left(\Delta m_{s} \cdot \sigma_{t}\right)^{2} / 2}$, with $P_{s}$ being the fraction of $B_{s}$ mesons in the sample, $\eta$ the probability to incorrectly tag whether the $B_{s}$ has mixed or not, and $\sigma_{t}$ the proper time resolution. From this formula it becomes obvious what the main ingredients of a $\Delta m_{s}$ analysis are:

For the mixing tag, the b-flavour has to be tagged at production and decay time. For the production tag, modern analyses combine information both from the opposite hemisphere (e.g. jet-charge, vertex-charge, leptons) and the same hemisphere (e.g. fragmentation charge, leading fragmentation particle). The decay tag and its performance are heavily depending on the channel under investigation (e.g. flavour of a reconstructed $D_{s}$ meson, charge of the lepton in semileptonic decays).

Precise reconstruction of the proper time $\boldsymbol{t}=\boldsymbol{m}_{\boldsymbol{B}} \boldsymbol{l} / \boldsymbol{p}$ is the most crucial point. Since $\sigma_{t} \approx \frac{m_{B}}{p} \sigma_{l} \oplus t \frac{\sigma_{p}}{p}$, sensitivity is given mainly to the first oscillations and the decay length resolution $\sigma_{l}$ - and thus precise reconstruction of the secondary decay vertex - becomes very important. The sample can be subdivided into resolution classes to profit from events with very good resolution being essential to gain sensitivity at high $\Delta m_{s}$.

Enrichment in $\boldsymbol{B}_{\boldsymbol{s}}$ mesons can be achieved by using e.g. vertex charge, charge of an inclusively reconstructed charm system or the fragmentation kaon expected to accompany a $B_{s}$ meson. For some analyses (e.g. exclusive $B_{s}$ or $D_{s}-X$ channels), this is given intrinsically.

$B_{s}$ oscillation results are presented in the framework of the 'Amplitude Method' [4i] simplifying the setting of limits and combination of analyses.

\section{Measurements at LEP}

Each LEP experiment collected about 4 million hadronic $\mathrm{Z}$ decays during the LEP I phase. Five basic analysis methods have been carried out by the LEP collaborations. Their specific strengths and weaknesses are summarised in Table 11.1.

ALEPH, L3 and OPAL didn't release new results for this conference. ALEPH performed analyses using exclusively reconstructed $B_{s}$ mesons, $D_{s}$-hadron and $D_{s}$-lepton cor-

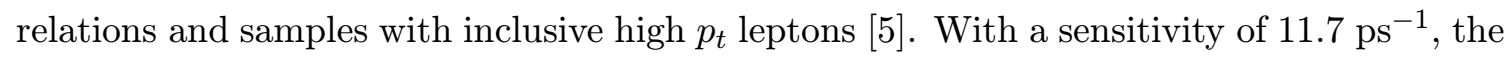
high $p_{t}$ lepton analysis is the most sensitive single measurement currently available ${ }^{1}$. OPAL analysed $D_{s}$-lepton and inclusive hight $p_{t}$ lepton events, giving sensitivities of $4.2 \mathrm{ps}^{-1}$ and $7.2 \mathrm{ps}^{-1}$, respectively [i]

\footnotetext{
${ }^{1}$ Some numbers quoted in this article might differ from the numbers in the original publication, because the $B$ Oscillations Working Group uses a common set of input variables which might differ from the one originally used by the collaboration in the publication.
} 


\begin{tabular}{|c||c||c|c|c|c|c|c|}
\hline Method & Collab. & $\mathrm{N}$ & $\sigma_{t}$ & $1-2 \eta$ & $P_{s}$ & sensitivity & $\sigma_{A}$ at high $\Delta m_{s}$ \\
\hline excl. $B_{s}$ & $\mathrm{AD}$ & $*$ & $* * * * *$ & $* * * * *$ & $* * * * *$ & $*$ & $* *$ \\
$D_{s}$-lepton & $\mathrm{ADO}$ & $* *$ & $* * * *$ & $* * * *$ & $* * * *$ & $* * * *$ & $* * * *$ \\
$D_{s}$-hadron & $\mathrm{AD}$ & $* * *$ & $* *$ & $* * *$ & $* * *$ & $* *$ & $*$ \\
incl. leptons & $\mathrm{ADO}$ & $* * * *$ & $* * *$ & $* * * *$ & $*$ & $* * * * *$ & $* * * * *$ \\
incl. vertices & $\mathrm{D}$ & $* * * * *$ & $*$ & $*$ & $*$ & $* * *$ & $* *$ \\
\hline
\end{tabular}

Table 1: Performances of the basic analysis methods performed by the LEP collaborations $(\mathrm{A}=\mathrm{ALEPH}, \mathrm{D}=\mathrm{DELPHI}, \mathrm{O}=\mathrm{OPAL})$, sorted in increasing order of the statistics of the samples. Marks are from one to five stars on a 'LEP scale', with a typical uncertainty of one star (depending on the specific methods and capabilities of the experiments etc.).

\subsection{New results from DELPHI}

Apart from the analysis using exclusively reconstructed $B_{s}$ mesons and $D_{s}$-hadron corre-

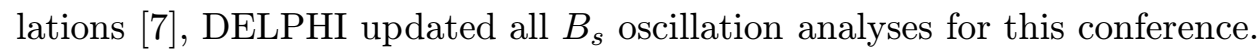

\subsubsection{Inclusive vertices and soft leptons [i8}

This analysis is based both on total inclusive vertex reconstruction and 'soft' leptons having a momentum $p>2 \mathrm{GeV}$ and not overlapping with the lepton selection of the analysis using 'high $p_{t}$ ' leptons $\left(p_{t}>1.2 \mathrm{GeV}\right)$. A B decay vertex is fitted in a fully inclusive way based on probabilities $P_{i}$ that a track stems from the secondary B decay vertex, defined by quantities like e.g. impact parameters and rapidity. The momentum estimate is also based on these probabilities and gives a momentum resolution of about $5 \mathrm{GeV}$. The sample is subdivided in eight resolution classes. The huge numbers of $130 \mathrm{k}$ and $500 \mathrm{k}$ events are available in the soft lepton and inclusive vertex sample, respectively. The production tag with a purity of about $71 \%$ is based on jet-charges for B decay and fragmentation particles as well as information from identified particles in the opposite hemisphere. In the same hemisphere, the fragmentation charge and identified particles (especially kaons) are used. For the soft leptons, the lepton-charge is used as decay tag. Additional variables like the momentum of the lepton in the B rest frame and its impact parameter with respect to the secondary vertex are exploited to separate between direct $b \rightarrow l$ and background $b \rightarrow c \rightarrow l$ decays, resulting in a purity of about $69 \%$. For the inclusive vertices, the decay tag is based on the reconstruction of the dipole charges (typically $W^{+}-D_{s}^{-}$in a $B_{s}$ decay) in the B rest frame, giving a purity of about $58 \%$.

This analysis excludes $\Delta m_{s}<1.1 \mathrm{ps}^{-1}$ and $\Delta m_{s} \in[1.5,5.3] \mathrm{ps}^{-1} @ 95 \% \mathrm{CL}$ and has a sensitivity of $6.1 \mathrm{ps}^{-1}$. The strength of this analysis is the large statistics of the samples leading to a competitive limit and sensitivity. However, due to the limited proper time resolution and the relatively poor decay tag (especially for the inclusive vertices), the error of the amplitude rapidly increases for high values of $\Delta m_{s}$.

\subsubsection{Inclusive leptons [9]}

Two algorithms are used for the reconstruction of the secondary decay vertex: the 'MiniJets Algorithm' finding the 'charm jet' from the B decay with a small $p_{t}$ based cluster 
parameter and the 'Grid Algorithm' testing candidate charm vertices around the 'B track'. The secondary B decay vertex is then obtained by intersecting the trajectories of a high $p_{t}$ lepton $\left(p_{t}>1.2 \mathrm{GeV}\right)$ and the charm candidate. A set of discriminant variables is used to choose the best solution among the algorithms and to define five decay length resolution classes, the best one having a resolution of about $180 \mu \mathrm{m}$. For the momentum estimate two algorithms are used as well and three resolution classes are defined, resulting in 15 proper time resolution classes. The production tag uses a multivariate neural-network based flavour-tag in the opposite hemisphere (using e.g. jet- and vertex charges and identified particles as input) and the fragmentation charge and possibly identified fragmentation kaon from the same hemisphere. To improve the decay tag, given by the charge of the lepton, two discriminant variables are used to enhance the fraction of $b \bar{b}$ events and the fraction of direct $b \rightarrow l$ decays. To enrich the sample in $B_{s}$ mesons, two discriminant variables are used (based on secondary vertex charge, number of tracks at the secondary vertex and charged kaons associated to primary and secondary vertex) to separate $B_{s}$ mesons from other neutral and charged $b$ hadrons. All these informations are used on event-by-event basis.

70k events are finally used. With a limit $\Delta m_{s}>11.7 \mathrm{ps}^{-1} @ 95 \% \mathrm{CL}$ and a sensitivity of $9.9 \mathrm{ps}^{-1}$, this is the most sensitive analysis from DELPHI.

\subsection{3 $D_{s}$-lepton correlations $[\overline{9}]$}

The published $D_{s}$-lepton analysis [1] ${ }_{1}^{1} \hat{O}_{1}^{\prime}$ already provided a good sensitivity of $8.1 \mathrm{ps}^{-1}$ (with a limit $\Delta m_{s}>7.4 \mathrm{ps}^{-1}$ ), but suffered from a rapid increase of the amplitude error at high $\Delta m_{s}$ because of the global parametrisation of $\sigma_{t}$. In the improved analysis presented here, $\sigma_{t}$ is used on event-by-event basis, isolating decays with excellent decay length resolution (based on variables like errors and $\chi^{2}$ of secondary and tertiary vertex fit) and momentum resolution (based on the reconstructed $\mathrm{B}$ momentum and $D_{s}$ mass). For the best events, resolutions of better than $200 \mu \mathrm{m}$ for $\sigma_{l}$ and $5 \%$ for $\sigma_{p} / p$ are achieved. So far, the channels $D_{s}^{-} \rightarrow \phi \pi^{-}$and $D_{s}^{-} \rightarrow K^{* 0} K^{-}$have been reanalysed with this technique in the $94 / 95$ data set (corresponding to about $30 \%$ of the total statistics), the other channels and years remaining unchanged.

This gives an increased sensitivity and limit of $8.7 \mathrm{ps}^{-1}$ and $7.9 \mathrm{ps}^{-1}$, respectively. However, the main improvement is the strongly decreased error of the amplitude at large $\Delta m_{s}$, e.g. at $\Delta m_{s}=20 \mathrm{ps}^{-1}, \sigma_{A}$ could be reduced by a factor of 1.3 .

\subsubsection{Combined DELPHI results}

Combination of all DELPHI $B_{s}$ oscillation results gives a limit of $\Delta m_{s}>14.5 \mathrm{ps}^{-1} @ 95 \%$ $\mathrm{CL}$ and a sensitivity of $12.0 \mathrm{ps}^{-1}$. Figures and the amplitude errors versus $\Delta m_{s}$ for all DELPHI analyses.

\section{Combined Results ['3ī]}

The compilation of oscillation results is performed by the $B$ Oscillations Working Group. Combination of the LEP results on $B_{s}$ oscillation gives $\Delta m_{s}>14.3 \mathrm{ps}^{-1} @ 95 \%$ CL with a 


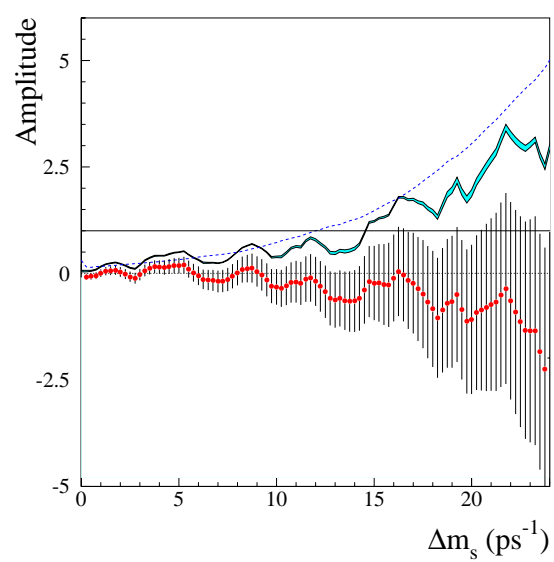

Figure 2: Combined amplitude distribution from DELPHI.

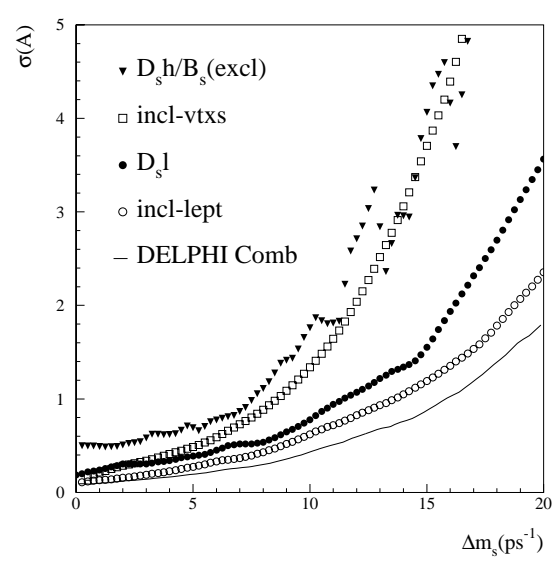

Figure 3: The error of the amplitude vs. $\Delta m_{s}$ for the DELPHI analyses.

sensitivity of $15.4 \mathrm{ps}^{-1}$. At last years summer conferences [1] 1 in 1 , the limit and sensitivity were $11.9 \mathrm{ps}^{-1}$ and $14.6 \mathrm{ps}^{-1}$, respectively. The gain is mainly due to the improved DELPHI analyses presented in this article.

Besides the LEP results, analyses on $B_{s}$ oscillations are available from SLD [1] $\left.12 \overline{1}\right]$ and CDF [13in. The world combined amplitude spectrum including also these results is shown in Figure The resulting limit is $\Delta m_{s}>14.6 \mathrm{ps}^{-1} @ 95 \%$ CL with a sensitivity of $18.3 \mathrm{ps}^{-1}$ (last year [1]1]1]: $15.0 \mathrm{ps}^{-1}$ and $18.1 \mathrm{ps}^{-1}$, respectively). The amplitude and its error at $\Delta m_{s}=17 \mathrm{ps}^{-1}$ are shown for all analyses in figure sensitivity, LEP and SLD roughly have equal weight.

The amplitude spectrum can be converted into a likelihood curve with respect to $\Delta m_{s}=\infty$ [i] to investigate the 'bump' at around $17 \mathrm{ps}^{-1}$. The minimum at $\Delta m_{s} \approx 17 \mathrm{ps}^{-1}$ has a significance of about 2.6 standard deviations.

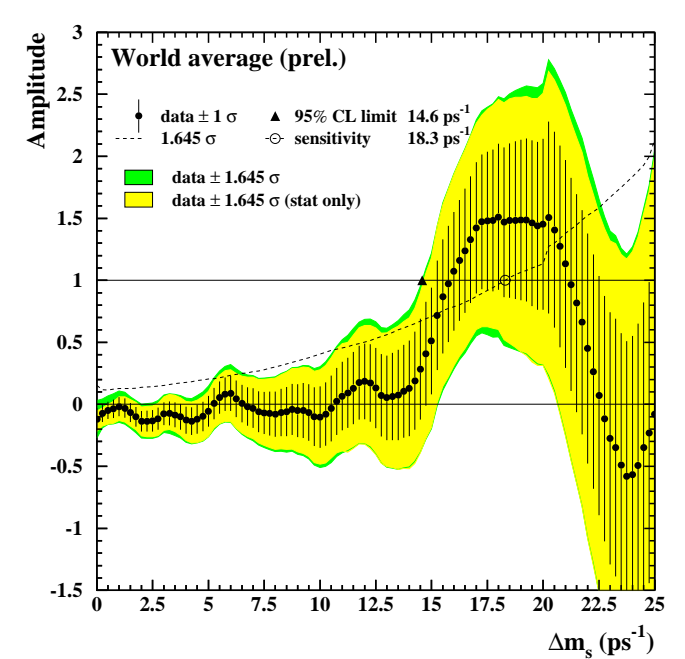

Figure 4: The combined $\mathrm{B}_{s}$ oscillation amplitude.

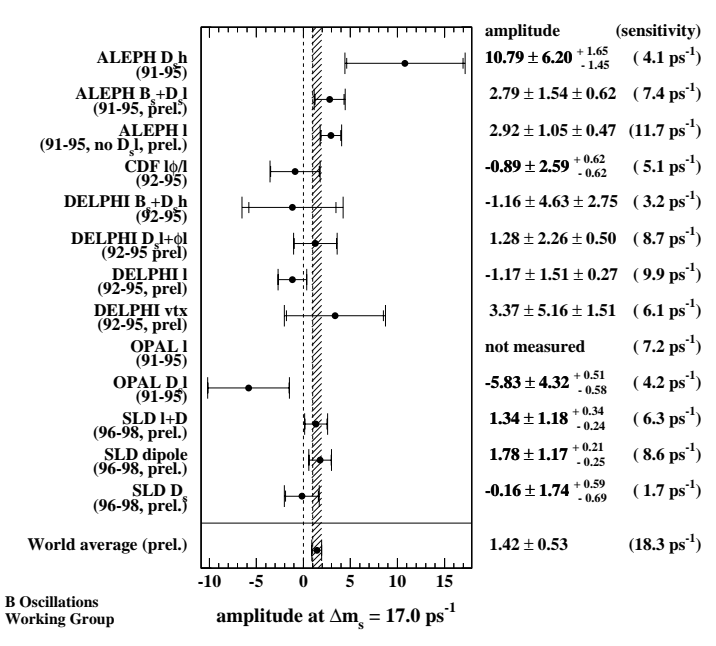

Figure 5: The amplitude and its error for all $\Delta m_{s}$ measurements at $\Delta m_{s}=17 \mathrm{ps}^{-1}$. 


\section{Conclusions and Outlook}

Impressive progress has been made in the field of $B_{s}$ oscillations in the last decade. The evolution of the sensitivity on $\Delta m_{s}$ with time is shown in Figure $i_{-i}^{i}$ Even six years after end of data-taking, there are still efforts to improve the analyses on $B_{s}$ oscillations at LEP and also SLD. However, it seems that these experiments are approaching their limits.

The current combined limits (and sensitivities) are $14.3 \mathrm{ps}^{-1}\left(15.4 \mathrm{ps}^{-1}\right)$ for LEP and $14.6 \mathrm{ps}^{-1}\left(18.3 \mathrm{ps}^{-1}\right)$ for all existing results.

These limits will be either improved significantly or $B_{s}$ mixing will be measured in the near future at the Tevatron in Run II (see e.g. [1]

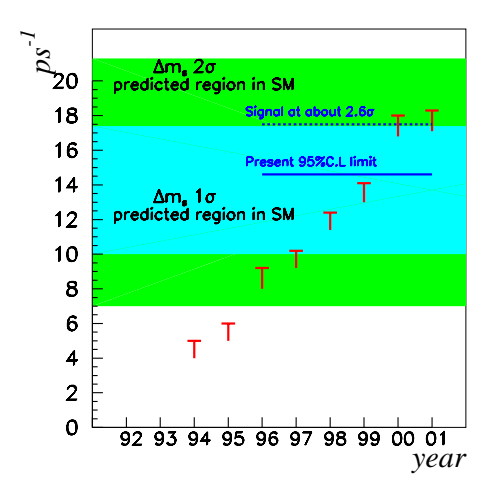

Figure 6: The evolution of the $\Delta m_{s}$ sensitivity in the last years.

\section{Acknowledgments}

I'd like to thank my colleagues Achille Stocchi and Fabrizio Parodi for fruitful discussion.

\section{References}

[1] M. Ciuchini et al., 'High Energy Phys. 07 (2001)

[2] L. Wolfenstein, 'Phys. Rev. Lett. 51 (1983) 1945!

[3] B Oscillations Working Group, Results for the summer 2001 conferences, http://lepbosc.web.cern.ch/LEPBOSC/combined_results/budapest_2001/

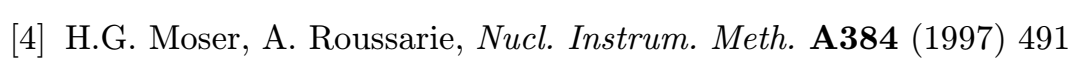

[5] ALEPH Collaboration, R. Barate et al., Eur. Phys. J.

ALEPH Collaboration, Contributed paper to ICHEP 2000,

ALEPH/2000-029 CONF/2000-024;

ALEPH Collaboration, Contributed paper to ICHEP 2000,

ALEPH/2000-059 CONF/2000-039

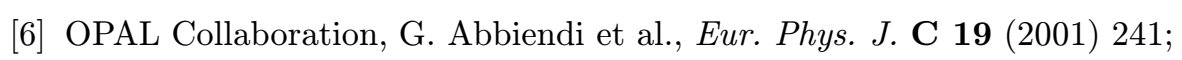

OPAL Collaboration, G. Abbiendi et al., Eur. Phys. J. 11 1999$) 58 \bar{T}_{1}$

[7] DELPHI Collaboration, P. Abreu et al., 'Eur. Phys.

[8] DELPHI Collaboration, T. Allmendinger et al., Contributed paper to EPS HEP 2001 and LP01, DELPHI 2001-054 CONF 482

[9] DELPHI Collaboration, P. Kluit et al., Contributed paper to EPS HEP 2001 and LP01, DELPHI 2001-055 CONF 483

[10] DELPHI Collaboration, P. Abreu et al., 'Eur. Phys. J. 16 $\mathbf{1}(2000) 555$

[11] ALEPH, CDF, DELPHI, L3, OPAL, SLD; CERN-EP/2001-50

[12] J. Thom, Time dependent $B^{0} \bar{B}^{0}$ Mixing at $S L D$, these proceedings

[13] CDF Collaboration, F. Abe et al., 'Phys. Rev. Lett. 82 (1999) 3576!

[14] A.B Wicklund, B Physics Prospects at Hadron Machines, these proceedings 